\title{
Pancreatic cyst fluid harboring a KRAS mutation
}

\author{
M. Rabie Al-Turkmani, Stuart R. Gordon, Kerrington D. Smith, \\ Gregory J. Tsongalis, and Xiaoying Liu
}

Department of Pathology and Laboratory Medicine and Department of Medicine, Dartmouth-Hitchcock Medical Center and Geisel School of Medicine, Lebanon, New Hampshire 03766, USA

Abstract A 55-yr-old woman presented with abdominal bloating for approximately 1 year. Imaging studies showed a cyst in the body of the pancreas with proximal pancreatic ductal dilation. An endoscopic ultrasound-guided fine-needle aspiration (EUS-FNA) was performed. Cytologic findings from the cyst fluid were consistent with a mucinous neoplastic cyst, and the possibility of malignancy could not be entirely excluded. A KRAS mutation analysis was performed on the cyst fluid using the Idylla system and circulating tumor KRAS (ctKRAS) cartridge (Biocartis, Mechelen, Belgium), which tests for actionable mutations in exons 2, 3, and 4 of the KRAS gene. Idylla testing detected a KRAS G12D mutation in the cyst fluid. The patient subsequently underwent a distal subtotal pancreatectomy with splenectomy. Microscopic examination of the resected tissue revealed an intraductal papillary mucinous neoplasm (IPMN) with an associated invasive carcinoma. KRAS testing on the resected tumor tissue confirmed the G12D mutation detected in the cyst fluid earlier. The described rapid testing of KRAS directly from the pancreatic cyst fluid can complement cytology assessment to classify pancreatic cysts more reliably and can potentially be of significant help when other cyst findings are nondiagnostic.

[Supplemental material is available for this article.]

\section{CASE PRESENTATION}

Corresponding author: m.rabie.al-turkmani @hitchcock.org

(c) 2019 Al-Turkmani et al. This article is distributed under the terms of the Creative Commons Attribution-NonCommercial

License, which permits reuse and redistribution, except for commercial purposes, provided that the original author and source are credited.

Ontology term: pancreatic cysts

Published by Cold Spring Harbor Laboratory Press

doi:10.1101/mcs.a003830
The patient is a 55-yr-old woman who has a past medical history that included a right mastectomy with contralateral prophylactic mastectomy (bilateral) in 2010. She was found to have a BRCA2 mutation, Y3308X, which she inherited from the paternal side of her family. This Y3308X mutation results in premature termination of the BRCA2 protein at amino acid 3308. Her father was diagnosed with a BRCA2 gene mutation and her paternal grandfather died of pancreatic cancer. She has several paternal aunts with breast cancer. On the maternal side of the family, her grandmother also died of pancreatic cancer.

The patient self-reported feeling bloated for approximately 1 year and a CT scan was ordered, which showed a cyst in the body of the pancreas with proximal pancreatic ductal dilation. Because of her BRCA2 status and the concern for pancreatic cancer (Mersch et al. 2015), an endoscopic ultrasound (EUS) was performed. An anechoic lesion suggestive of a cyst was identified in the pancreatic body and it communicated with the pancreatic duct that was moderately dilated upstream $(3.5 \mathrm{~mm}$ ) (Fig. 1A). The lesion measured $29 \mathrm{~mm}$ by $22 \mathrm{~mm}$ in maximal cross-sectional diameter. There were a few compartments thickly septated. The outer wall of the lesion was thin. There was no associated mass. There was no internal debris within the fluid-filled cavity. An endoscopic ultrasound-guided fine-needle aspiration (EUS-FNA) was performed and the fluid was clear and watery. Samples were sent for amylase 
A

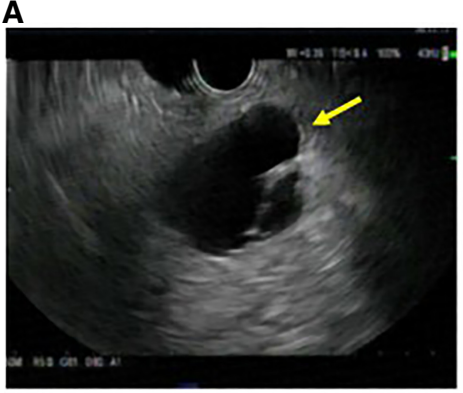

D

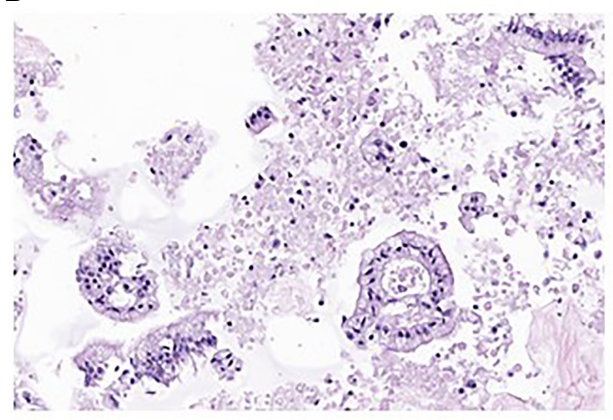

$\mathbf{F}$

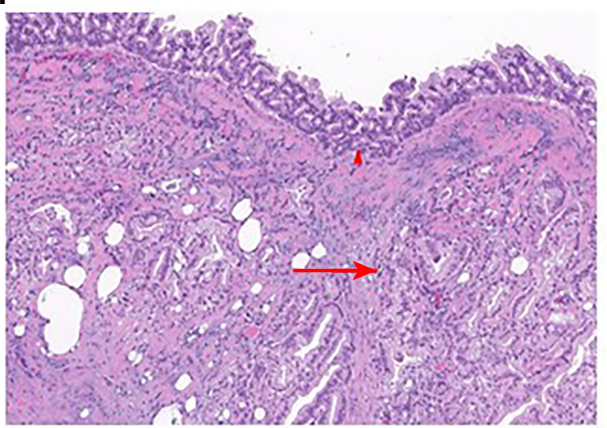

B

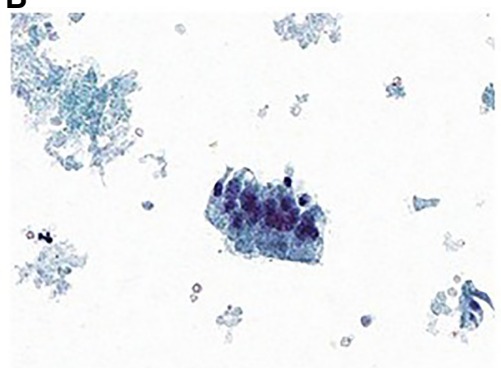

E

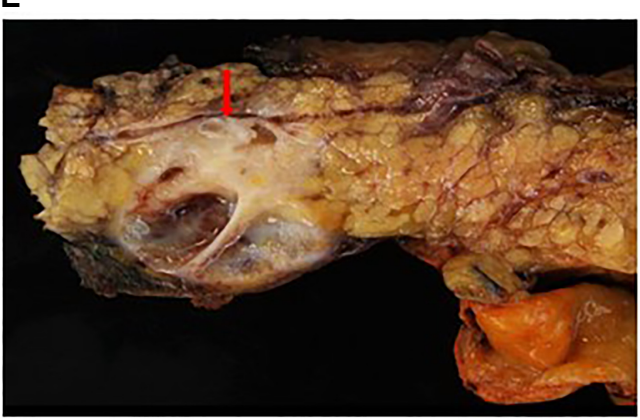

G

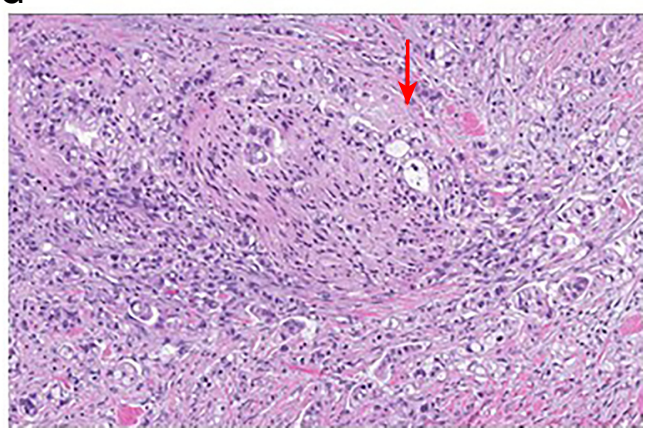

Figure 1. (A) Endoscopic ultrasound (EUS) showing a 29-mm septated cystic lesion seen in the pancreatic body, associated with upstream ductal dilation. The outer wall of the lesion was thin. There was no associated mass. (B) Mucinous/gastric foveolar-type epithelial cells in a small cluster. (C) Focal cytologic and architecture atypia and background necrosis. (D) Cell block showing a few clusters of mucinous/gastric foveolar-type epithelial cells and background necrosis. (E) Cyst at the body of pancreas measuring $4.1 \times 2.2 \times 2.0 \mathrm{~cm}$ with solid area extending into the pancreatic duct (arrow). (F) IPMN (arrowhead) with an associated invasive carcinoma (arrow). (G) Invasive carcinoma with perineural invasion (arrow).

and carcinoembryonic antigen (CEA) testing as well as cytopathologic examination. There was no sign of significant endosonographic abnormality in the common bile duct. There was an 8-mm stone in the gallbladder. No lymphadenopathy was seen.

On cytologic examination, single and clusters of mucinous/gastric foveolar-type epithelial cells were present (Fig. 1B). This was consistent with a mucinous neoplastic cyst. Focal cytologic atypia with possible necrosis was seen, and the possibility of malignancy could not be entirely excluded (Fig. 1C). The cyst fluid amylase was 66 units/L and the CEA was $8334 \mathrm{ng} / \mathrm{mL}$.

The patient denied any nausea, anorexia symptoms, or early satiety. She initially had no weight loss but later started showing dyspepsia-like symptoms and lost nearly 10 pounds. There were no symptoms of pancreatic exocrine insufficiency or pancreatitis. Based on the 
cyst size, its relatively thick septations, and atypical features on cytology, she was referred to surgery for resection.

A distal subtotal pancreatectomy with splenectomy was performed, which showed a $4.1 \times 2.2 \times 2 \mathrm{~cm}$ cyst in the body of the pancreas. The cyst had a solid area extending into the pancreatic duct (Fig. 1E). Microscopic examination revealed an intraductal papillary mucinous neoplasm (IPMN) with an associated invasive carcinoma (Fig. 1F). Perineural invasion was present (Fig. 1G). The tumor invaded peripancreatic soft tissues, and all margins were uninvolved by invasive carcinoma. All examined lymph nodes were negative for carcinoma.

\section{TECHNICAL ANALYSIS AND METHODS}

A $100-\mu \mathrm{L}$ aliquot of the pancreatic cyst fluid was analyzed shortly after EUS-FNA using the Idylla system (Biocartis, Mechelen, Belgium), which is a cartridge-based molecular system that provides automated sample processing and targeted mutation detection directly from formalin-fixed, paraffin-embedded (FFPE) tissue or plasma samples, with all reagents included in a single-use cartridge. The specific cartridge assay used for molecular analysis of the cyst fluid was the ctKRAS assay, which is designed to detect KRAS mutations in circulating tumor DNA using $1 \mathrm{~mL}$ of plasma directly added to the cartridges. In our case, we directly added cyst fluid to the cartridge. The cartridge tests for 21 mutations in exons 2, 3 , and 4 of the KRAS gene and the analytical sensitivity is $\leq 1 \%$ for mutations in exons 2 and 3 and $\leq 5 \%$ for mutations in exon 4 (Supplemental Table S1). During testing, DNA liberation, PCR amplification, and detection occur in the cartridge for a total run time of $\sim 2 \mathrm{~h}$. Additional KRAS testing on the resected tumor tissue was performed 3 mo after cyst fluid testing using one 10- $\mu \mathrm{m}$ FFPE tissue section and the Idylla KRAS cartridge, which tests tissue sections for the same KRAS mutations as the ctKRAS cartridge.

The Idylla assays use a real-time PCR chemistry based on PlexPrime and PlexZyme (also known as MNAzyme) technology (Mokany et al. 2010). With this technology, each primer is designed to have a $5^{\prime}$ target-recognition region, a short $3^{\prime}$ target-specific sequence complementary to the mutation of interest, and a distinct insert sequence that is mismatched to the target. This results in the production of allele-specific amplicons that are detected in real time by allele-specific PlexZyme enzymes and a universal fluorescent probe, allowing for detection of multiple mutations in a single multiplex reaction. Several studies have been performed on the Idylla system using cancer specimens from different tissues and they showed excellent concordance with other available molecular methods, including next-generation sequencing (Al-Turkmani et al. 2018; Uguen and Troncone 2018).

\section{VARIANT INTERPRETATION}

The Idylla testing detected a KRAS G12D (c.35G>A) mutation in the pancreatic cyst fluid. This single-nucleotide mutation in codon 12 of exon 2 results in replacement of the GGT codon for glycine with the GAT codon for aspartic acid, leading to impairment of the intrinsic GTPase activity of RAS and subsequent activation of downstream signaling pathways that drive cancer growth (Table 1). Subsequent KRAS testing of the resected tumor tissue confirmed the G12D mutation detected earlier in the pancreatic cyst fluid.

Table 1. Variant table

\begin{tabular}{cccccc}
\hline Gene & Chromosome & HGVS DNA & HGVS protein & Variant type & Predicted effect \\
\hline KRAS & 12 & c.35G $>$ A & p.G12D & Substitution & Impairment of GTPase activity \\
\hline
\end{tabular}


Pancreatic cancer is the fourth leading cause of cancer death in the United States, and it is expected to be the second by 2030 (Rahib et al. 2014). The activating point mutation of the KRAS oncogene in codon 12 is present in up to $94 \%$ of pancreatic ductal adenocarcinoma (PDAC) and it appears to be the initiating genetic event for this malignancy (Waters and Der 2018). PDAC patients harboring the KRAS G12D mutation, the same mutation detected in the pancreatic cyst fluid and tumor tissue here, were found to have a significantly shorter overall survival compared with other patients with wild-type KRAS or other codon 12 KRAS mutations (Bournet et al. 2016).

Many of the pancreatic cystic neoplasms, including serous cystadenomas, are benign and can be monitored periodically. In contrast, IPMNs and mucinous cystic neoplasms (MCNs) have the potential to progress to invasive pancreatic adenocarcinoma. Recently, DNA-based testing has emerged as an adjunct test to the assessment of pancreatic cysts. In a prospective, multicenter study that included 113 patients with 40 malignant, 48 premalignant, and 25 benign pancreatic cysts, KRAS mutation was helpful in identifying mucinous cysts with $96 \%$ specificity (Khalid et al. 2009). Another more recent study on 52 pancreatic cyst fluids classified as mucinous (40\%) or nonmucinous (60\%) found that cysts harboring KRAS mutations were more commonly mucinous and malignant (Faias et al. 2018). An additional study on 626 pancreatic cyst fluid samples that were tested preoperatively by NGSidentified KRAS/GNAS mutations in $49 \%$ of the samples, and these mutations were highly sensitive for IPMNs and specific for mucinous pancreatic cysts (Singhi et al. 2018). Combining KRAS/GNAS mutations to alteration in TP53/PIK3CA/PTEN, present in $6 \%$ of cases, was a useful preoperative marker for advanced neoplasia. Based on these studies, detection of KRAS mutations can complement cytology assessment in order to more reliably detect malignant mucinous lesions.

\section{SUMMARY}

Direct, rapid mutation analysis of KRAS in pancreatic cyst fluid was achieved in the presented case using the Idylla ctKRAS Mutation Assay. The detected KRAS G12D mutation further supported the mucinous nature of the cyst. Such KRAS analysis can be of significant help in identifying mucinous cysts when cyst morphology and cytology are nondiagnostic. The high sensitivity of the Idylla assay and the minimal amount of sample required for testing make it particularly helpful for cases where cellular content and fluid volume of pancreatic cysts are suboptimal for other routine ancillary studies, such as cytopathologic examination and CEA quantitation.

Unlike most molecular methods currently available for somatic mutation testing, Idylla testing can be performed directly on body fluids, plasma, or FFPE tissue sections without the need for sample preprocessing or extraction. The system also allows for random access testing, which results in rapid turnaround times. Additionally, Idylla requirements for space and infrastructure are minimal, allowing for its placement outside the central laboratory for expedited testing process and timely patient management decisions. A limitation of the Idylla testing is that it does not provide mutation allele fraction (MAF), which would provide further insight into the neoplastic cellularity of the tumor. Another limitation is the limited number of KRAS mutations the ctKRAS cartridge tests for and the lack of assays that test for mutations in other genes that play a key role in the development and progression of pancreatic cancer including CDKN2A, SMAD4, and TP53.

To our knowledge, this is the first United States-based report describing the Idylla ctKRAS testing and its use with pancreatic cyst fluids. Further studies comparing pancreatic cyst fluid results by the Idylla to those obtained from concurrent testing of the cell block or resected tumor tissue would help establish the analytical sensitivity and specificity of the 
Idylla ctKRAS assay. Our study offers a proof of concept and promise of providing simple and fast KRAS mutation analysis in pancreatic cyst fluids.

\section{ADDITIONAL INFORMATION}

\section{Data Deposition and Access}

The described variant has been submitted to ClinVar (http://www.ncbi.nlm.nih.gov/clinvar/) and can be found under accession number SVC000882700.

\section{Ethics Statement}

The study was approved by the Committee for the Protection of Human Subjects at Dartmouth-Hitchcock Medical Center, and the results obtained were not used for diagnostic purposes of any kind.

\section{Acknowledgments}

We thank the staff of the Laboratory for Clinical Genomics and Advanced Technology (CGAT) at Dartmouth-Hitchcock Medical Center.

Competing Interest Statement

The authors have declared no competing interest.

Received November 30, 2018; accepted in revised form February 1, 2019.

\section{Author Contributions}

All authors contributed to scientific discussion and manuscript review.

\section{Funding}

This work was funded by the Department of Pathology at Dartmouth-Hitchcock Medical Center.

\section{REFERENCES}

Al-Turkmani MR, Godwin KN, Peterson JD, Tsongalis GJ. 2018. Rapid somatic mutation testing in colorectal cancer by use of a fully automated system and single-use cartridge: a comparison with next-generation sequencing. J Appl Lab Med 3: 178-184. doi:10.1373/jalm.2018.026278

Bournet B, Muscari F, Buscail C, Assenat E, Barthet M, Hammel P, Selves J, Guimbaud R, Cordelier P, Buscail L. 2016. KRAS G12D mutation subtype is a prognostic factor for advanced pancreatic adenocarcinoma. Clin Transl Gastroenterol 7: e157. doi:10.1038/ctg.2016.18

Faias S, Duarte M, Albuquerque C, da Silva JP, Fonseca R, Roque R, Dias Pereira A, Chaves P, Cravo M. 2018. Clinical impact of KRAS and GNAS analysis added to CEA and cytology in pancreatic cystic fluid obtained by EUS-FNA. Dig Dis Sci 63: 2351-2361. doi:10.1007/s10620-018-5128-y

Khalid A, Zahid M, Finelstein SD, LeBlanc JK, Kaushik N, Ahmad N, Brugge WR, Edmundowicz SA, Hawes RH, McGrath KM. 2009. Pancreatic cyst fluid DNA analysis in evaluating pancreatic cysts: a report of the PANDA study. Gastrointest Endosc 69: 1095-1102. doi:10.1016/j.gie.2008.07.033

Mersch J, Jackson MA, Park M, Nebgen D, Peterson SK, Singletary C, Arun BK, Litton JK. 2015. Cancers associated with BRCA1 and BRCA2 mutations other than breast and ovarian. Cancer 121: 269-275. doi:10 $.1002 / \mathrm{cncr} .29041$

Mokany E, Bone SM, Young PE, Doan TB, Todd AV. 2010. MNAzymes, a versatile new class of nucleic acid enzymes that can function as biosensors and molecular switches. J Am Chem Soc 132: 1051-1059. doi:10.1021/ja9076777

Rahib L, Smith BD, Aizenberg R, Rosenzweig AB, Fleshman JM, Matrisian LM. 2014. Projecting cancer incidence and deaths to 2030: the unexpected burden of thyroid, liver, and pancreas cancers in the United States. Cancer Res 74: 2913-2921. doi:10.1158/0008-5472.CAN-14-0155

Singhi AD, McGrath K, Brand RE, Khalid A, Zeh HJ, Chennat JS, Fasanella KE, Papachristou GI, Slivka A, Bartlett DL, et al. 2018. Preoperative next-generation sequencing of pancreatic cyst fluid is highly accurate 
in cyst classification and detection of advanced neoplasia. Gut 67: 2131-2141. doi:10.1136/gutjnl-2016313586

Uguen A, Troncone G. 2018. A review on the Idylla platform: towards the assessment of actionable genomic alterations in one day. J Clin Pathol 71: 757-762. doi:10.1136/jclinpath-2018-205189

Waters AM, Der CJ. 2018. KRAS: the critical driver and therapeutic target for pancreatic cancer. Cold Spring Harb Perspect Med 8: a031435. doi:10.1101/cshperspect.a031435 


\section{COLD SPRING HARBOR Molecular Case Studies}

\section{Pancreatic cyst fluid harboring a KRAS mutation}

M. Rabie Al-Turkmani, Stuart R. Gordon, Kerrington D. Smith, et al.

Cold Spring Harb Mol Case Stud 2019, 5: a003830

Access the most recent version at doi: $10.1101 /$ mcs.a003830

\section{Supplementary http://molecularcasestudies.cshlp.org/content/suppl/2019/03/25/mcs.a003830.D} Material C1

References This article cites 10 articles, 5 of which can be accessed free at: http://molecularcasestudies.cshlp.org/content/5/2/a003830.full.html\#ref-list-1

License This article is distributed under the terms of the Creative Commons Attribution-NonCommercial License, which permits reuse and redistribution, except for commercial purposes, provided that the original author and source are credited.

Email Alerting Receive free email alerts when new articles cite this article - sign up in the box at the Service top right corner of the article or click here. 